\title{
Supplementary Information for Temporal and state abstractions for efficient learning, transfer and composition in humans
}

\author{
Liyu Xia, Anne G. E. Collins
}

\section{Potential asymmetry in Block 7 of Experiment 1}

We checked whether the performance of circle and square in Block 7 was asymmetrically affected due to the interleaving of odd and even blocks (Fig. 2B). Specifically, participants might start Block 7 by using $H O_{1}$ in odd blocks; thus the negative transfer in the first stage of Block 7 would be primarily due to more key presses from the square, not the circle.

To test this possibility, we calculated average number of key presses in the first 5 trials for circle and square respectively in Block 7. However, we found no significant difference between the performance of circle and square in the first stage (paired t-test, $t(24)=1.38, p=0.18$ ); we also found no significant difference between the performance in the second stage following circle and square (paired t-test, $t(24)=0.44, p=0.66$ ).

\section{Second stage reaction time and sequence learning effects}

Sequence learning predicts that the reaction time of the "sequence" type to be faster than the "non-sequence" type. Therefore, we calculated the average reaction time (Supplementary Fig. S1, Supplementary Fig. S2) for both "sequence" and "non-sequence" error types in Experiment 1 and 2.

\section{Experiment 1}

We broke down each block to 2 different time periods: early (trials 1-7 for each of the 4 branches in the second stage) and late (trials 8-15 for each of the 4 branches). Aggregating Blocks 3-6, we found a marginal effect of time period (2-way repeated measure ANOVA, $F(1,21)=3.0, p=0.099$ ), which might be due to participants generally becoming faster as they progressed within a block. We also found a main effect of error type (2-way repeated measure ANOVA, $F(1,21)=4.5, p=0.046)$ on reaction time. Specifically, we found no significant difference $(t(23)=1.3, p=0.2)$ between the reaction time of the "sequence" 

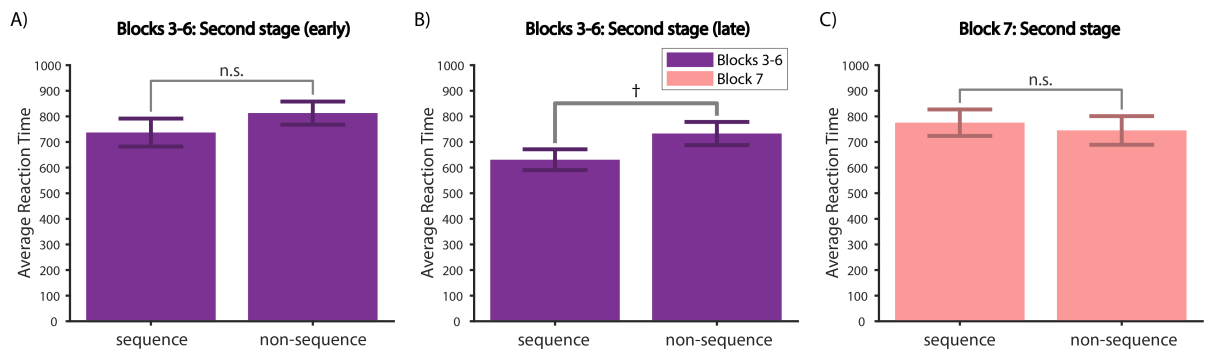

Supplementary Figure S1: Experiment 1 reaction time. (A) Average reaction time for trials 1-7 for each of the 4 branches in the second stage for Blocks 3-6 for sequence (left) and non-sequence (right) error types. (B) Same as (A) for trials 8-15. (C) Average reaction time for sequence (left) and non-sequence (right) error types in the second stage of Block 7.

and "non-sequence" error types in the early time periods (Supplementary Fig. $\mathrm{S} 1 \mathrm{~A}$ ). The "sequence" type was marginally faster (paired t-test, $t(22)=1.9, p=$ 0.072 ) than the "non-sequence" type in the late time period (Supplementary Fig. $\mathrm{S} 1 \mathrm{~B}$ ). We also found no significant difference (paired t-test, $t(20)=1.1, p=$ 0.3 ) between the "sequence" and "non-sequence" types in the entire Block 7 (Supplementary Fig. S1C). These results suggest that the transfer effects we observed at the beginning of each block could not be due to pure sequence learning, which only start to take effect during learning saturation.

\section{Experiment 2}

A)

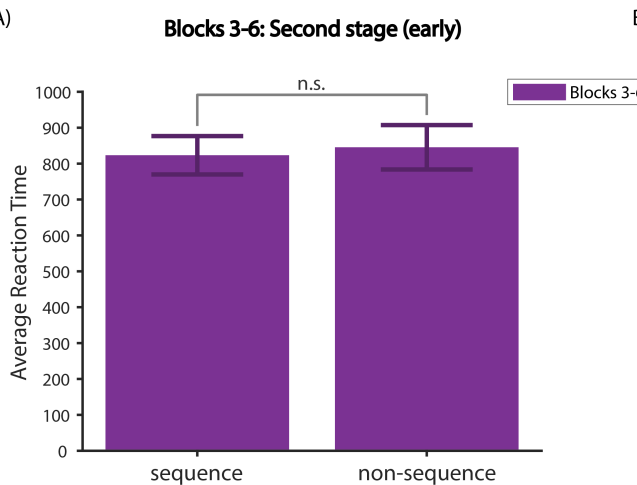

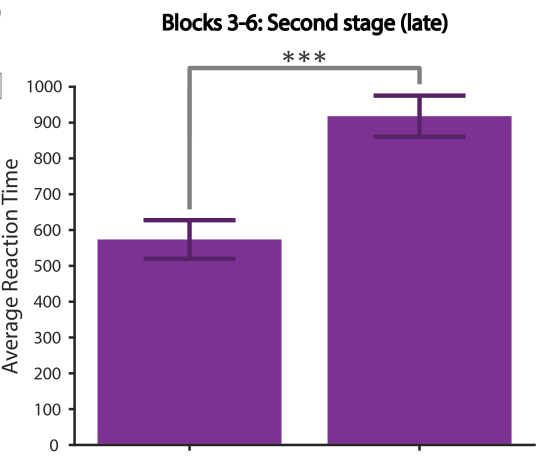

sequence

non-sequence

Supplementary Figure S2: Experiment 2 reaction time. (A) Average reaction time for trials 1-4 for each of the 4 branches in the second stage for Blocks 3-6 for sequence (left) and non-sequence (right) error types. (B) Same as (A) for trials $5-8$.

We also analyzed the reaction time (Supplementary Fig. S2) of the "se- 
quence" and "non-sequence" error types in Blocks 5-6 in Experiment 2. As in Experiment 1, we broke down each block into 2 halved time periods: early (trials 1-4 for each of the 4 branches in the second stage) and late (trials 5-8 for each of the 4 branches). We found a main effect of time period and error type, and a significant interaction (2-way repeated measure ANOVA, time period: $F(1,16)=8, p=0.012$; error type: $F(1,16)=16, p=0.0009$; interaction: $F(1,16)=15, p=0.0013)$. Specifically, there was no significant difference (Supplementary Fig. S2A) between the reaction time of the "sequence" and "nonsequence" types in the early time period (paired t-test, $t(21)=0.61, p=0.55$ ). However, the "sequence" type was significantly faster (Fig. S2B) than the "nonsequence" type in the late period (paired t-test, $t(17)=4.8, p=0.0002$ ). These results replicated the trend observed in the second stage of Experiment 1 (Supplementary Fig. S1): sequence learning might take effect during learning saturation, but not the beginning of blocks, where we typically expect to observe transfer effects.

\section{Full description of the Option Model}

The first stage of the Option Model is identical to the first stage of the Task-Set Model. The model tracks the probability $P^{1}$ of selecting each first stage taskset $H O_{i}$ in different first stage contexts $c_{j}^{1}$, which encodes the current temporal (block) context (e.g. 8 contexts in the first stage of Experiment 1 due to 8 blocks). The model uses CRP to select $H O$ : if contexts $\left\{c_{1: n}^{1}\right\}$ are clustered on $N^{1} \leq n H O$ s, when the model encounters a new context $c_{n+1}^{1}$, the prior probability of selecting a new high-level option $H O_{n+1}$ in this new context is set to:

$$
P^{1}\left(H O_{n+1} \mid c_{n+1}^{1}\right)=\frac{\gamma^{1}}{Z^{1}}
$$

and the probability of reusing a previously created high-level option $H O_{i}$ is set to:

$$
P^{1}\left(H O_{i} \mid c_{n+1}^{1}\right)=\frac{N_{i}^{1}}{Z^{1}}
$$

where $\gamma^{1}$ is the clustering coefficient for the CRP, $N_{i}^{1}$ is the number of first stage contexts clustered on $H O_{i}$, and $Z^{1}=\gamma^{1}+\sum_{i} N_{i}^{1}$ is the normalization constant. The new $H O_{n+1}$ policy is initialized with uninformative Q-values $1 / \#\{$ possible actions $\}=\frac{1}{4}$. The model samples $H O$ based on the conditional distribution over all $H O$ s given the current temporal context. The model also tracks $H O$-specific policies via Q-learning. Once an $H O$ is selected, a first stage policy is computed based on the HO's Q-values and the first stage stimulus $F_{i}$ with softmax:

$$
P\left(A_{j}^{1} \mid F_{i}, H O\right)=\frac{\exp \left(\beta^{1} * Q_{H O}^{1}\left(F_{i}, A_{j}^{1}\right)\right)}{\sum_{k} \exp \left(\beta^{1} * Q_{H O}^{1}\left(F_{i}, A_{k}^{1}\right)\right)},
$$

where $\beta^{1}$ is the inverse temperature. A first stage action $A^{1}$, ranging from $A_{1}$ to $A_{4}$, is then sampled from this softmax policy. After observing the outcome 
(moving on to the second stage or not), the model uses Bayes' Theorem to update $P^{1}$ :

$$
P^{1}\left(H O_{k} \mid c_{j}^{1}\right)=\frac{P\left(r \mid F_{i}, A^{1}, H O_{k}\right) P\left(H O_{k} \mid c_{j}^{1}\right)}{\left(\sum_{l} P\left(r \mid F_{i}, A^{1}, H O_{l}\right) P\left(H O_{l} \mid c_{j}^{1}\right)\right)},
$$

where $r$ is 1 if $A^{1}$ is correct and 0 otherwise, and $P\left(r \mid F_{i}, A^{1}, H O_{l}\right)=1-$ $Q_{H O_{l}}^{1}\left(F_{i}, A^{1}\right)$ if $r=0$, or $Q_{H O_{l}}^{1}\left(F_{i}, A^{1}\right)$ if $r=1$. Then the Q-values of the $H O$ with the highest posterior probability is updated:

$$
Q_{H O}^{1}\left(F_{i}, A^{1}\right)=Q_{H O}^{1}\left(F_{i}, A^{1}\right)+\alpha^{1} *\left(r-Q_{H O}^{1}\left(F_{i}, A^{1}\right)\right),
$$

where $\alpha^{1}$ is the learning rate. After each choice, the model decays the Q-values of each $\mathrm{HO}$ in the first stage based on $f^{1}$ :

$$
Q_{H O}^{1}\left(F_{i}, A_{j}^{1}\right)=\left(1-f^{1}\right) * Q_{H O}^{1}\left(F_{i}, A_{j}^{1}\right)+f^{1} * \frac{1}{4} .
$$

Forgetting in the second stage is implemented similarly.

The second stage is similar to the second stage of the Task-Set Model. The only difference is that each $M O$ has an $M O$-specific probability table $P_{M O}^{2}$. In the Task-Set Model, the CRP in the second stage using $P^{2}$ is independent of the first stage choices. In contrast, in the Option Model, the first stage choice determines which $M O$ is activated (e.g. choosing $A_{1}$ for the circle in Experiment 1 is equivalent to choosing $M O_{1}$ as a whole, Fig. 2A), which then determines which probability table, $P_{M O}^{2}$, to use for running the CRP in the second stage and to select $L O \mathrm{~s}$. This implementation captures the essence of options in the HRL framework, in that selection of $M O$ in the first stage constrains the policy chosen until the end of the second stage (where the option terminates). Specifically, for the $P_{M O}^{2}$ activated by the $M O$ chosen in the first stage, there are 16 contexts in the second stage of Experiment 1 ( 8 blocks and 2 first stage stimuli). If contexts $\left\{c_{1: n}^{2}\right\}$ are clustered on $N^{2} \leq n L O \mathrm{~s}$, when the model encounters a new context $c_{n+1}^{2}$, the prior probability of selecting a new low-level option $L O_{n+1}$ in this new context is set to:

$$
P_{M O}^{2}\left(L O_{n+1} \mid c_{n+1}^{2}\right)=\frac{\gamma^{2}}{Z^{2}}
$$

and the probability of reusing a previously created low-level option $L O_{i}$ is set to:

$$
P_{M O}^{2}\left(L O_{i} \mid c_{n+1}^{2}\right)=\frac{N_{i}^{2}}{Z^{2}},
$$

where $\gamma^{2}$ is the clustering coefficient for the CRP, $N_{i}^{2}$ is the number of second stage contexts clustered on $L O_{i}$ for the current $M O$, and $Z^{2}=\gamma^{2}+\sum_{i} N_{i}^{2}$ is the normalization constant. The new $L O_{n+1}$ policy is initialized with uninformative Q-values $1 / \#\{$ possible actions $\}=\frac{1}{4}$. The model samples $L O$ based on the conditional distribution over all LOs given the current context and $M O$. The model also tracks $L O$-specific policies via Q-learning. Once an $L O$ is selected, 
a second stage policy is computed based on the $L O$ 's Q-values and the second stage stimulus $S_{i}$ with softmax:

$$
P\left(A_{j}^{2} \mid S_{i}, L O\right)=\frac{\exp \left(\beta^{2} * Q_{L O}^{2}\left(S_{i}, A_{j}^{2}\right)\right)}{\sum_{k} \exp \left(\beta^{2} * Q_{L O}^{2}\left(S_{i}, A_{k}^{2}\right)\right)},
$$

where $\beta^{2}$ is the inverse temperature. To account for the meta-learning heuristic, we add a free meta-learning parameter, $m$, that discourages selecting the same action in the second stage as in the first stage. Specifically, if $\pi$ is the second stage policy as computed from softmax, we set $P\left(A^{1}\right)=m$, where $A^{1}$ is the action chosen in the first stage, and re-normalize:

$$
P\left(A^{\text {other }}\right)=(1-m) \times \pi\left(A^{\text {other }}\right) /\left(1-\pi\left(A^{1}\right)\right),
$$

where $A^{\text {other }}$ is any action other than $A^{1}$. A second stage action $A^{2}$, ranging from $A_{1}$ to $A_{4}$, is then sampled from this policy. After observing the outcome (moving on to the second stage or not), the model uses Bayes' Theorem to update $P_{M O}^{2}$ :

$$
P_{M O}^{2}\left(L O_{k} \mid c_{j}^{2}\right)=\frac{P\left(r \mid S_{i}, A^{2}, L O_{k}\right) P_{M O}^{2}\left(L O_{k} \mid c_{j}^{2}\right)}{\left(\sum_{l} P\left(r \mid S_{i}, A^{2}, L O_{l}\right) P_{M O}^{2}\left(L O_{l} \mid c_{j}^{2}\right)\right)},
$$

where $r$ is 1 if $A^{2}$ is correct and 0 otherwise, and $P\left(r \mid S_{i}, A^{2}, L O_{l}\right)=1-$ $Q_{L O_{l}}^{2}\left(S_{i}, A^{2}\right)$ if $r=0$, or $Q_{L O_{l}}^{2}\left(S_{i}, A^{2}\right)$ if $r=1$. Then the Q-values of the $L O$ with the highest posterior probability is updated:

$$
Q_{L O}^{2}\left(S_{i}, A^{2}\right)=Q_{L O}^{2}\left(S_{i}, A^{2}\right)+\alpha^{2} *\left(r-Q_{L O}^{2}\left(S_{i}, A^{2}\right)\right),
$$

where $\alpha^{2}$ is the learning rate.

\section{Parameters for model simulations}

\section{Parameters used for main text}

We used the set of parameters from Supplementary Table S1 in the main text to track participants' behavioral patterns both qualitatively and quantitatively.

\section{A set of constrained parameters that capture behavior across all tasks qualitatively}

In the main text, we selected parameters to try to trace participants' behavior patterns both quantitatively and qualitatively (Supplementary Table S1). Here we used another set of parameters (Supplementary Table S2) to (1) constrain parameters so that most experiments shared the same parameters while showing the qualitatively trends in participants' behavior and (2) show that the model can reproduce the same qualitative effects with a range of parameters. 


\begin{tabular}{|c|c|c|c|c|c|c|c|c|c|c|c|}
\hline Exp & Sample & Model & $\alpha^{1}$ & $\beta^{1}$ & $\gamma^{1}$ & $f^{1}$ & $\alpha^{2}$ & $\beta^{2}$ & $\gamma^{2}$ & $f^{2}$ & $\mathrm{~m}$ \\
\hline \multirow{4}{*}{ Exp 1 } & \multirow{4}{*}{ In-lab } & Naive & 0.5 & 4 & NA & 0.0025 & 0.7 & 10 & NA & 0.0001 & 0.01 \\
\cline { 3 - 13 } & & Flat & 0.5 & 4 & NA & 0.0025 & 0.7 & 10 & NA & 0.0001 & 0.01 \\
\cline { 3 - 13 } & & Task-Set & 1 & 2 & 14 & 0.0004 & 0.8 & 3 & 3 & 0.0002 & 0.01 \\
\cline { 3 - 12 } & Option & 1 & 2 & 14 & 0.0004 & 0.8 & 3 & 3 & 0.0002 & 0.01 \\
\hline & Mturk & Option & 0.8 & 3 & 100 & 0.01 & 0.6 & 6 & 5 & 0.004 & 0.01 \\
\hline Exp 2 & In-lab & Option & 0.7 & 3 & 13 & 0.001 & 0.6 & 4 & 5 & 0.001 & 0.01 \\
\hline \multirow{2}{*}{ Exp 3 } & In-lab & Option & 0.7 & 4 & 100 & 0.01 & 0.8 & 5 & 15 & 0.001 & 0.01 \\
\cline { 2 - 12 } & Mturk & Option & 0.7 & 4 & 100 & 0.01 & 0.8 & 5 & 15 & 0.005 & 0.01 \\
\hline \multirow{3}{*}{ Exp 4 } & In-lab & Option & 0.6 & 4 & 100 & 0.01 & 0.8 & 5 & 4 & 0.0002 & 0.01 \\
\cline { 3 - 11 } & \multirow{2}{*}{ Mturk } & Option & 0.6 & 4 & 100 & 0.01 & 0.4 & 4 & 5 & 0.002 & 0.01 \\
\cline { 3 - 11 } & & Task-Set & 0.6 & 4 & 100 & 0.01 & 0.4 & 4 & 5 & 0.002 & 0.01 \\
\hline
\end{tabular}

Supplementary Table S1: Parameters for the main text.

\begin{tabular}{|c|c|c|c|c|c|c|c|c|c|c|c|}
\hline Exp & Sample & Model & $\alpha^{1}$ & $\beta^{1}$ & $\gamma^{1}$ & $f^{1}$ & $\alpha^{2}$ & $\beta^{2}$ & $\gamma^{2}$ & $f^{2}$ & $\mathrm{~m}$ \\
\hline \multirow{4}{*}{ Exp 1 } & \multirow{3}{*}{ In-lab } & Naive & 0.7 & 4 & NA & 0.001 & 0.7 & 4 & NA & 0.001 & 0.01 \\
\cline { 3 - 12 } & & Flat & 0.7 & 4 & NA & 0.001 & 0.7 & 4 & NA & 0.001 & 0.01 \\
\cline { 3 - 12 } & & Task-Set & 0.7 & 4 & 14 & 0.001 & 0.7 & 4 & 4 & 0.001 & 0.01 \\
\cline { 3 - 12 } & Oturk & Option & 0.7 & 4 & 14 & 0.001 & 0.7 & 4 & 4 & 0.001 & 0.01 \\
\hline \multirow{2}{*}{ Exp 2 } & In-lab & Option & 0.7 & 4 & 100 & 0.01 & 0.5 & 4 & 4 & 0.005 & 0.01 \\
\hline \multirow{2}{*}{ Exp 3 } & In-lab & Option & 0.7 & 4 & 100 & 0.01 & 0.7 & 4 & 4 & 0.001 & 0.01 \\
\cline { 2 - 12 } & Mturk & Option & 0.7 & 4 & 100 & 0.01 & 0.7 & 4 & 20 & 0.001 & 0.01 \\
\hline \multirow{2}{*}{ Exp 4 4} & In-lab & Option & 0.7 & 4 & 100 & 0.01 & 0.7 & 4 & 4 & 0.001 & 0.01 \\
\cline { 2 - 11 } & Mturk & Option & 0.7 & 4 & 100 & 0.01 & 0.5 & 4 & 4 & 0.005 & 0.01 \\
\hline
\end{tabular}

Supplementary Table S2: A second set of parameters that is constrained but still replicate transfer effects qualitatively.

In particular, we used $\alpha^{1}=0.7, \beta^{1}=4, \beta^{2}=4, m=0.01$ for all experiments. For all in-lab experiments, we used $\alpha^{2}=0.7, f^{2}=0.001$; for all Mturk experiments, we used $\alpha^{2}=0.5, f^{2}=0.005$, which indicate slower learning rate and faster forgetting. For Experiment 1 in-lab, we used $\gamma^{1}=14, f^{1}=0.001$; for all other experiments, we used $\gamma^{1}=100, f^{1}=0.01$ to implement a lack of transfer effects in the first stage. We used $\gamma^{2}=20$ in Experiment 3 to model reduced option transfer in the second stage; for all other experiments, we used $\gamma^{2}=4$.

We recreated some of the representative analysis in the main text to demonstrate that this second set of parameters can replicate the transfer effects in human participants qualitatively well (Supplementary Fig. S3, Supplementary Fig. S4, Supplementary Fig. S5). 
A)

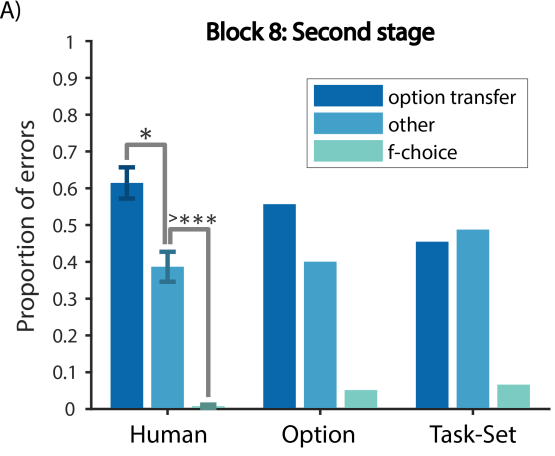

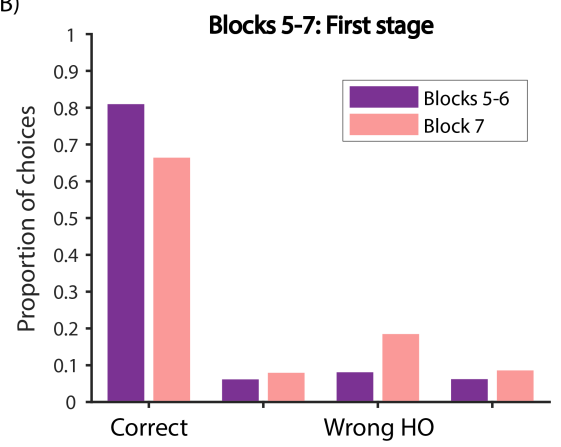

Supplementary Figure S3: Experiment 1 with parameters from Supplementary Table S2. (A) Error type analysis of the second stage in Block 8 for participants (left), the Option Model (middle) and the Task-Set Model (right). (B) Choice type analysis of the first stage in Blocks 5-7 for the Option Model.

A)

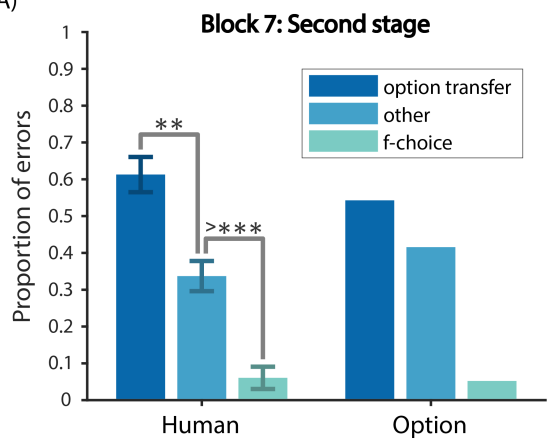

B)

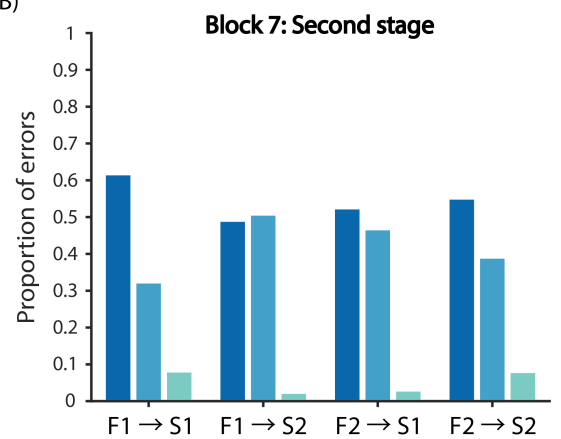

Supplementary Figure S4: Experiment 2 second stage choices with parameters from Supplementary Table S2 (A) Error type analysis of the second stage in Block 7 for participants (left) and the Option Model (right). (B) Error type analysis for each of the 4 branches in the second stage of Block 7 for the Option Model. 

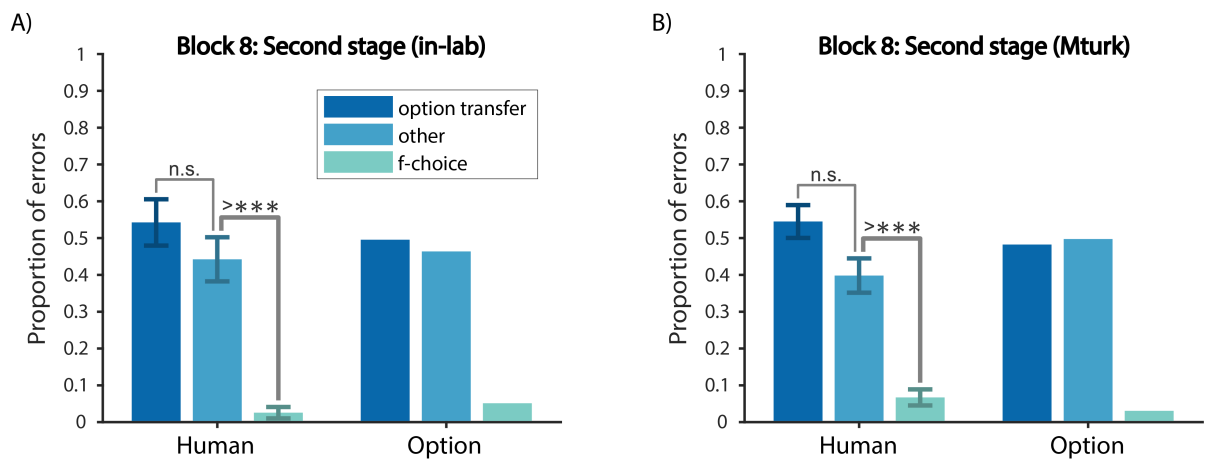

Supplementary Figure S5: Experiment 3 second stage choices with parameters from Supplementary Table S2. Error type analysis of the second stage in Block 8 for (A) in-lab participants (left) and the Option Model (right), and (B) Mturk participants (left) and the Option Model (right).

A)

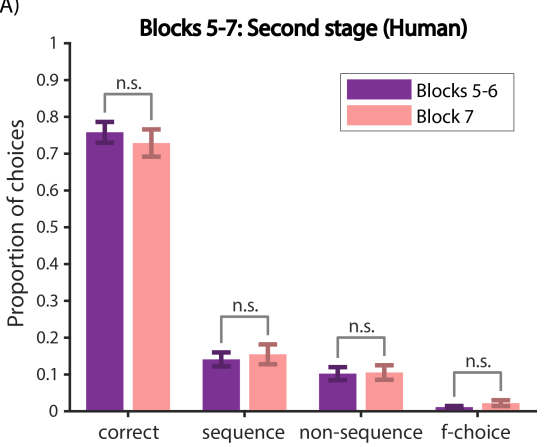

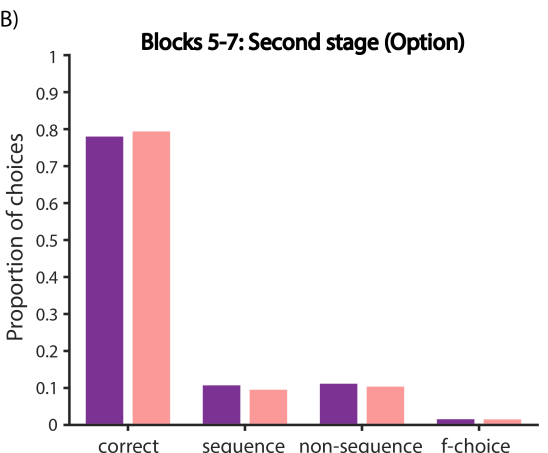

Supplementary Figure S6: Experiment 1 second stage choices. Choice type analysis of the second stage comparing Blocks 5-6 and Block 7 for (A) participants and (B) the Option Model. There was no significant difference across all choice types, indicating positive transfer in the second stage of Block 7 . 


\section{First stage}

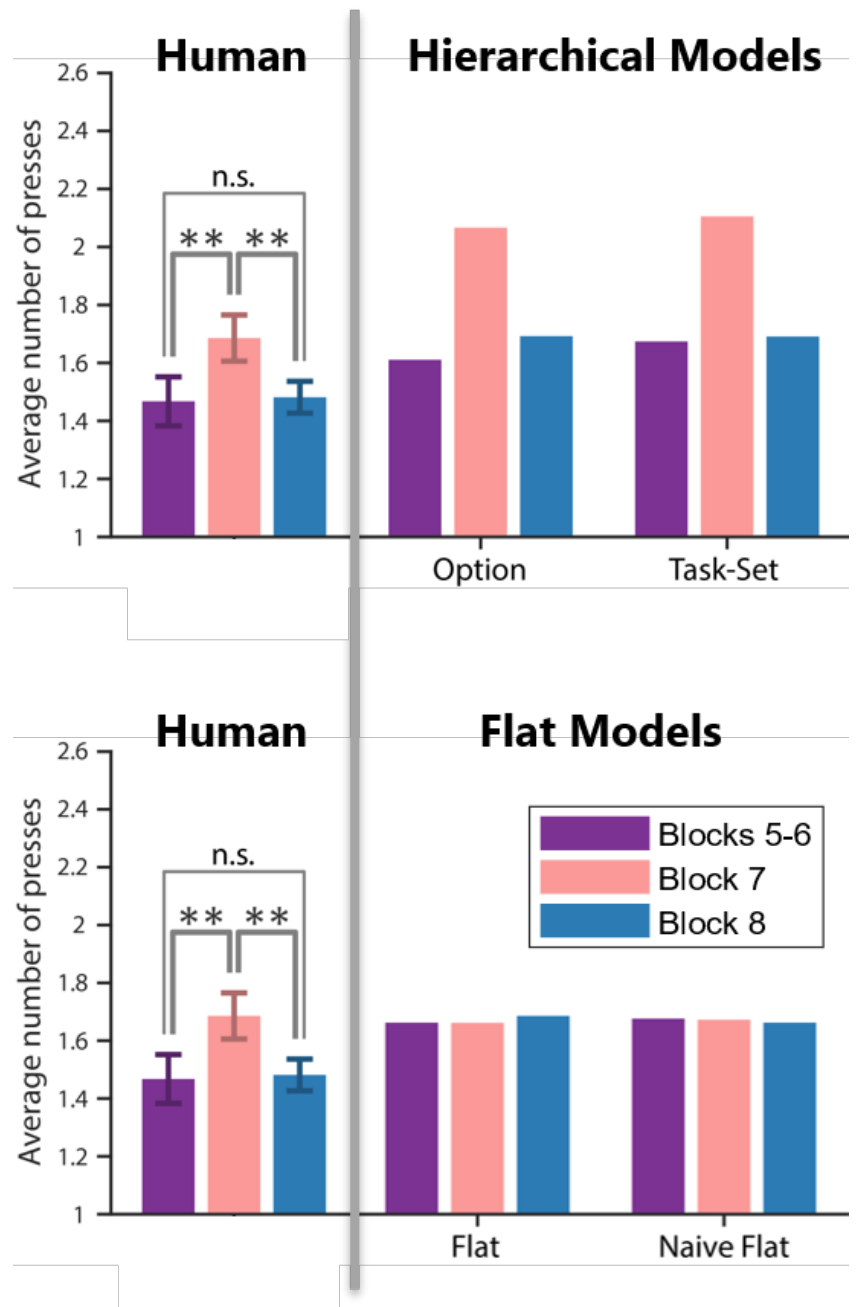

Supplementary Figure S7: Experiment 1 first stage transfer effects. Average number of first stage key presses in the first 10 trials of Block 5-8 for participants as well as model simulations. We ran 500 simulations of each hierarchical model (top) and flat model (bottom). See Supplementary Table S1 for model parameters. Behavioral results show patterns of positive and negative transfer predicted by hierarchical, but not flat RL models. 

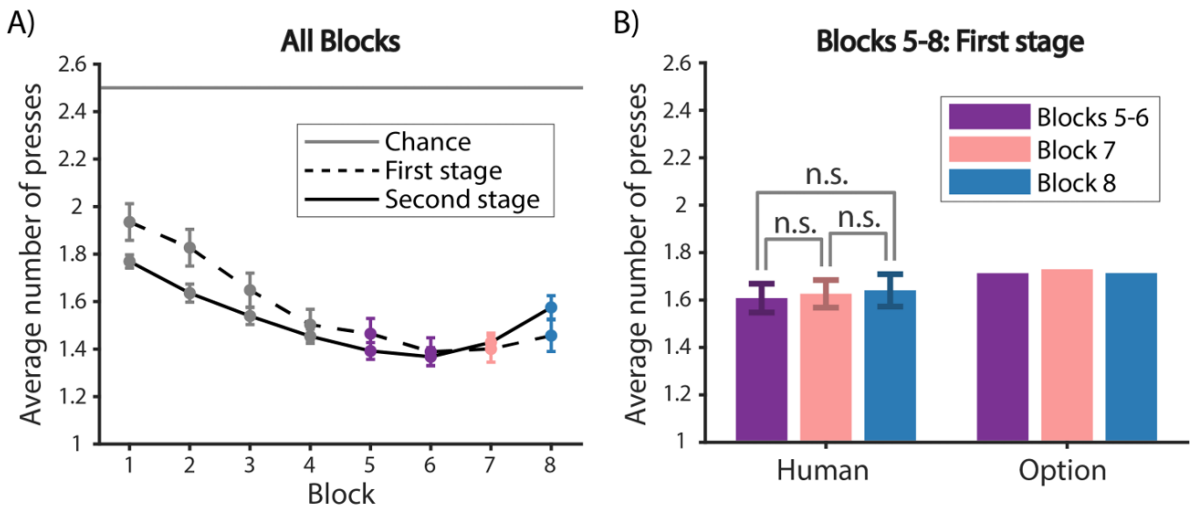

Supplementary Figure S8: Experiment 1 Mturk results. (A) Average number of key presses in the first and the second stages per block. (B) Average number of key presses for the first 10 trials of Blocks 5-8 for the first stage for participants (left) and the Option Model (right).

A)

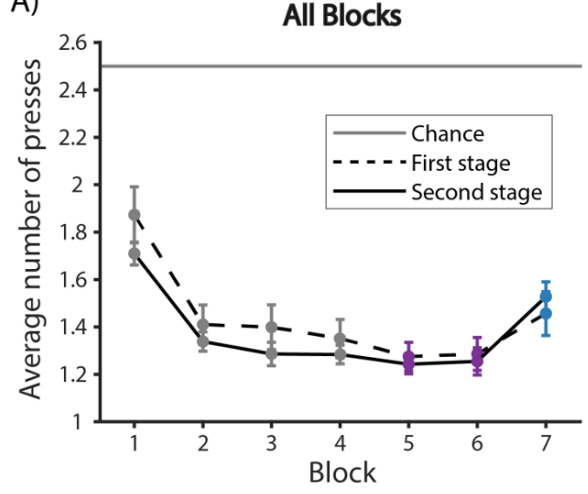

B)

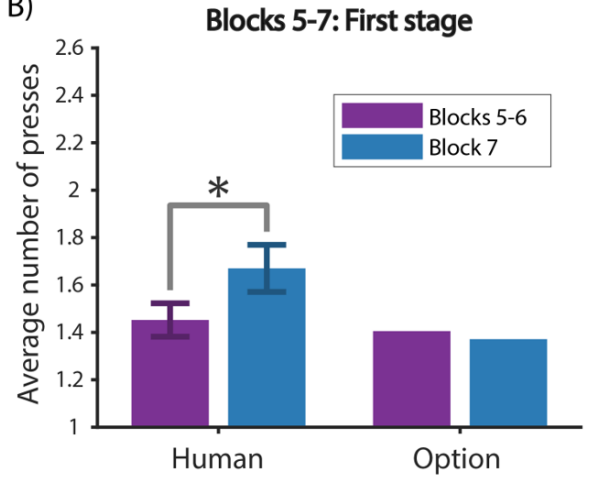

Supplementary Figure S9: Experiment 2 results. (A) Average number of key presses in the first and the second stages per block. (B) Average number of key presses for the first 10 trials of Blocks 5-7 for the first stage for participants (left) and the Option Model (right). 


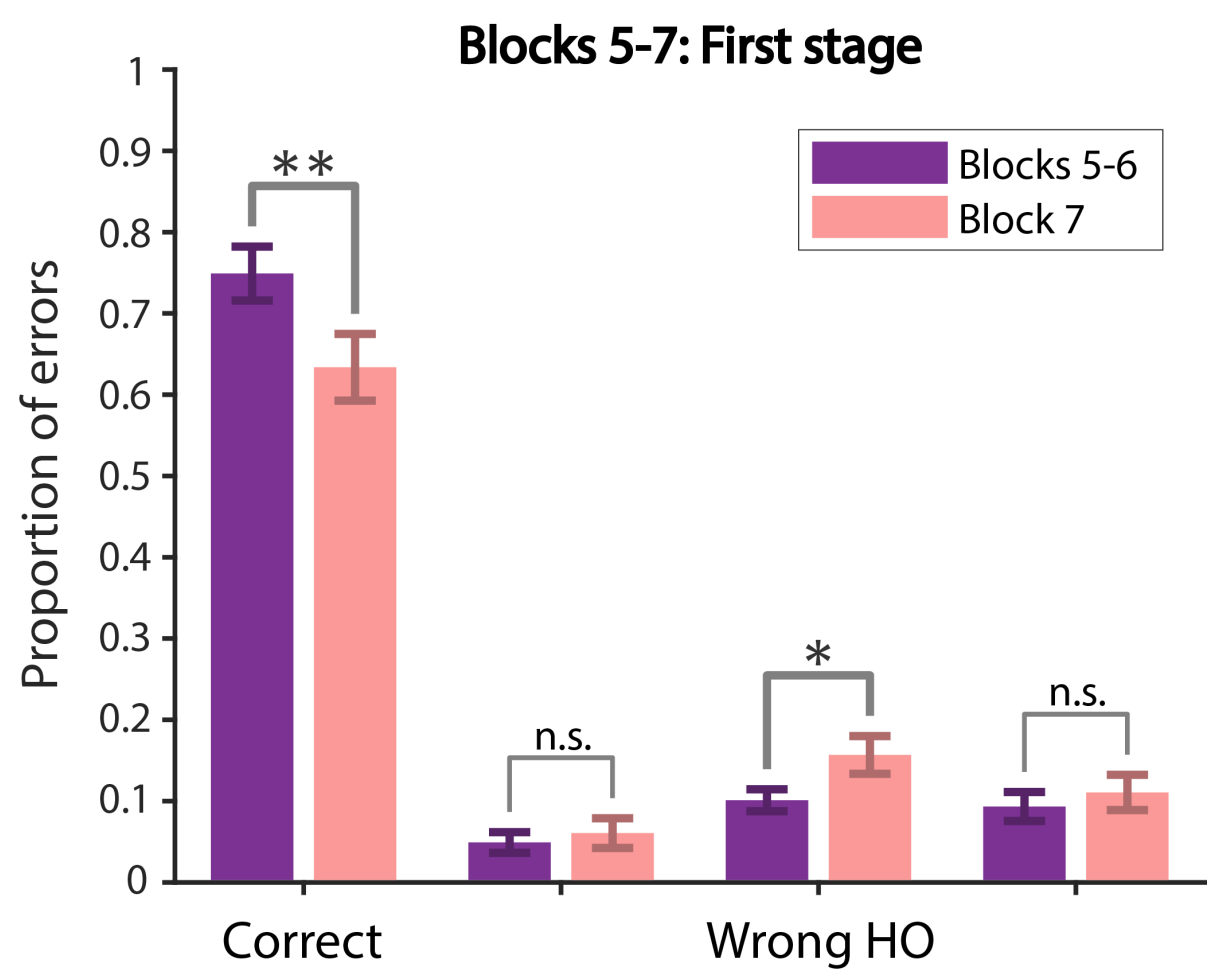

Supplementary Figure S10: Experiment 2 first stage choices. Choice type analysis of the first stage comparing Blocks 5-6 and Block 7. The only error type that significantly increased was the wrong $\mathrm{HO}$ error, suggesting that participants were perseverating in the first stage while learning the new mappings in the second stage of Block 7. 

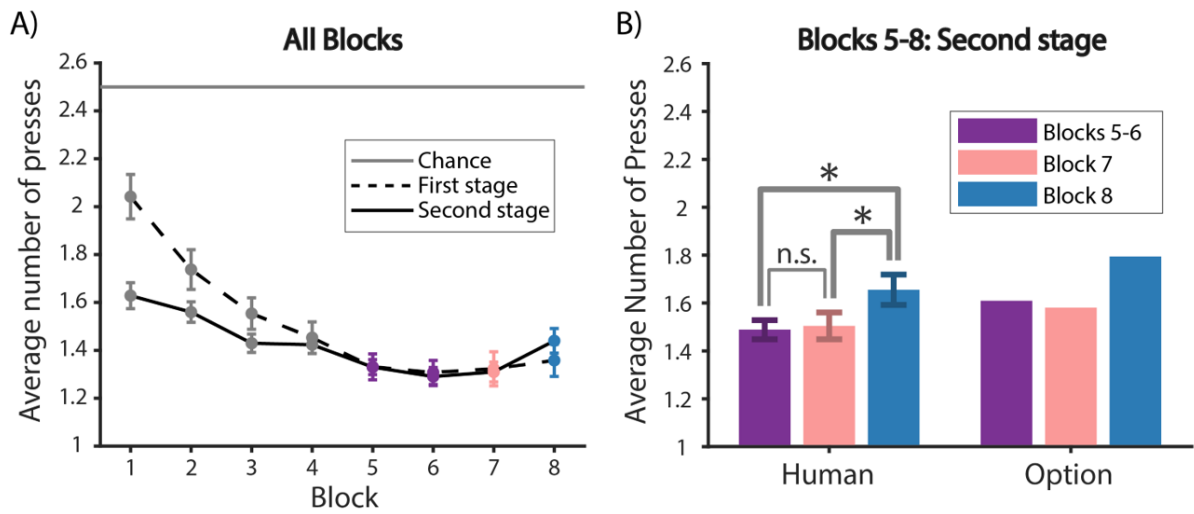

Supplementary Figure S11: Experiment 3 Mturk results. (A) Average number of key presses in the first and the second stages per block. (B) Average number of key presses for the first 10 trials of Blocks 5-8 for the second stage for participants (left) and the Option Model (right). 


\section{Blocks 5-8: First stage}

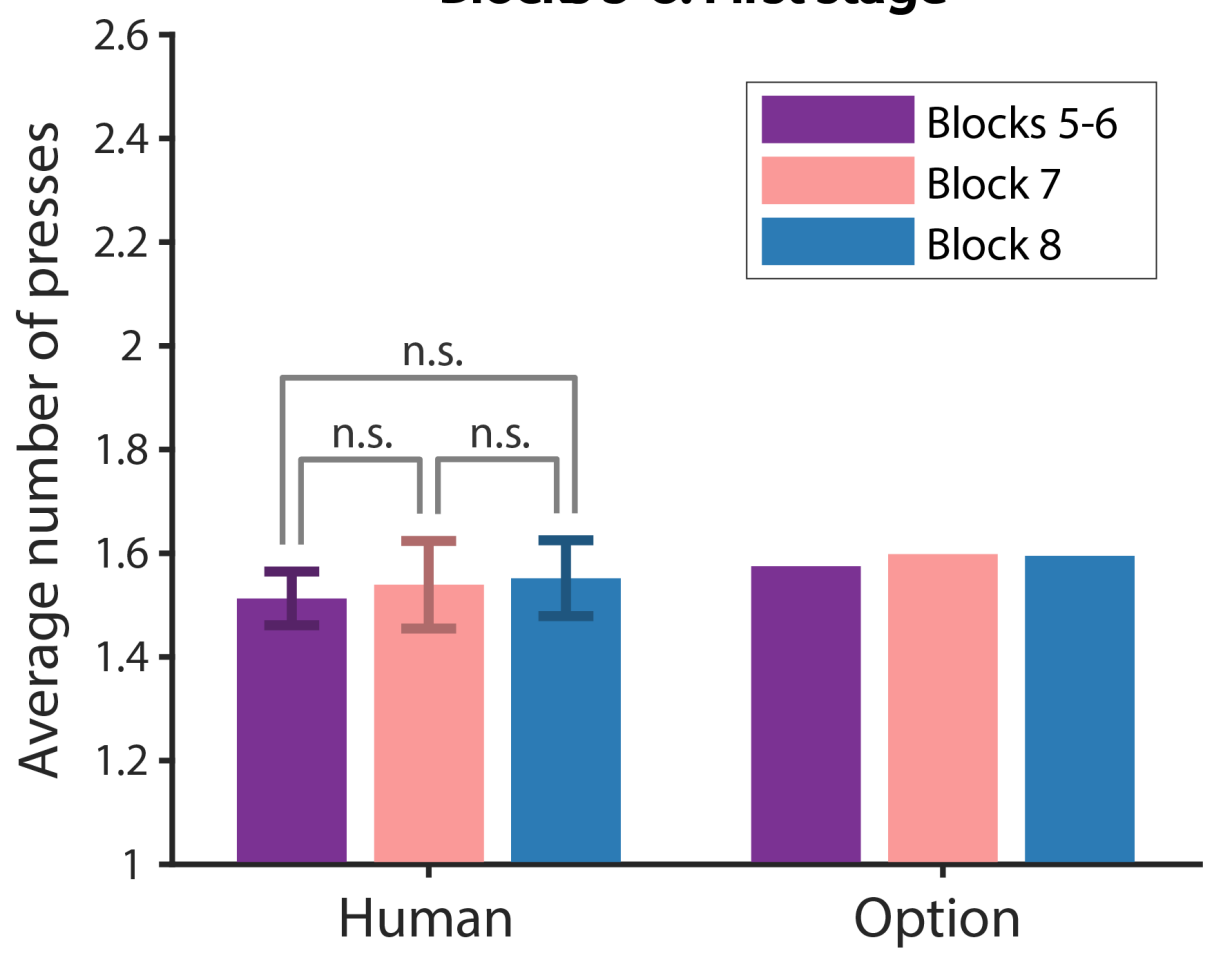

Supplementary Figure S12: Experiment 3 Mturk first stage choices. Average number of presses in the first 10 trials of Blocks 5-8 in the first stage for participants (left) and the Option Model (right). This shows a lack of transfer in the first stage, representative of Experiments 3-4 first stage for both in-lab and Mturk populations. 

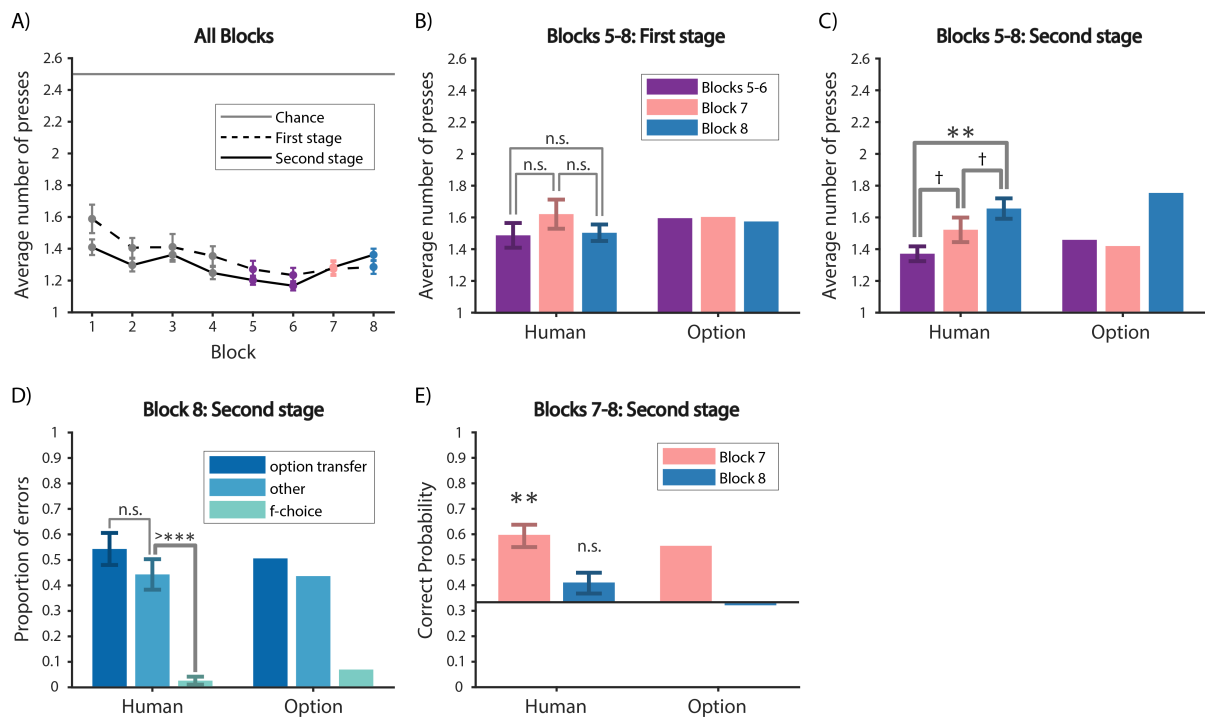

Supplementary Figure S13: Experiment 3 summary. (A) Average number of key presses in the first and the second stages per block. (B) Average number of key presses for the first 10 trials of Blocks 5-8 for the first stage for participants (left) and the Option Model (right). (C) Same as (B) for the second stage. (D) Error type analysis of the second stage in Block 8 for participants (left) and the Option Model (right). The proportion of option transfer error was not significantly different from other error, different from Experiment 1 and Experiment 2, suggesting reduced option transfer. (E) Probability of a correct first key press for the second stage of the first trial of each of the 4 branches in Blocks 7-8 for participants (left) and the Option Model (right).

A)

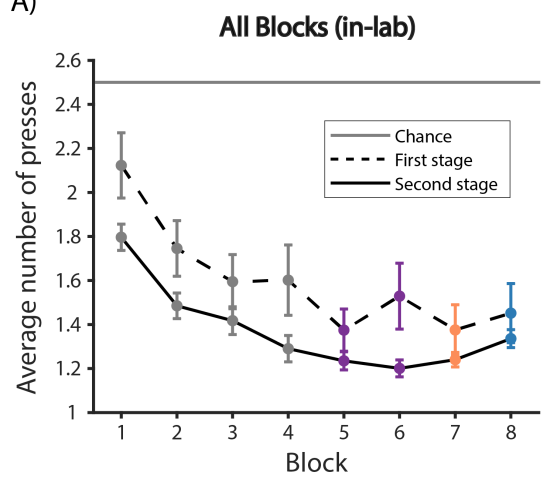

B)

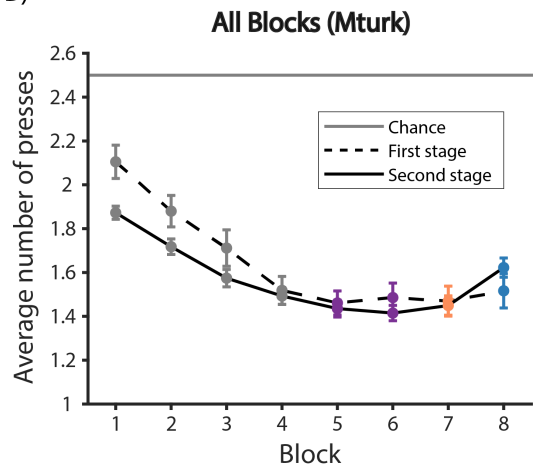

Supplementary Figure S14: Experiment 4 number of presses. Average number of key presses in the first and the second stages per block for (A) in-lab participants and (B) Mturk participants. 


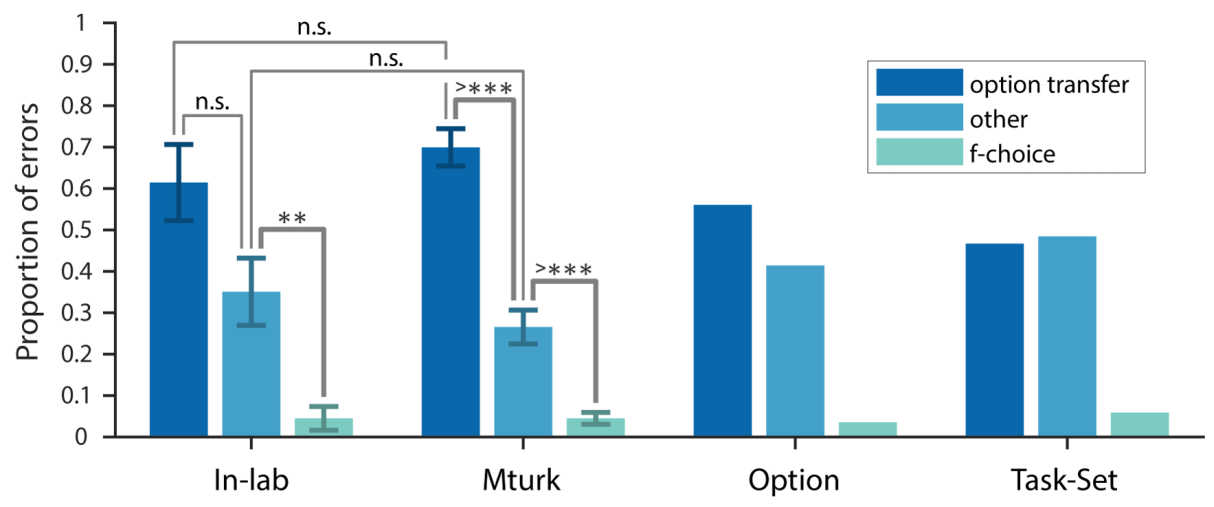

Supplementary Figure S15: Experiment 4 second stage errors reveal temporal options transfer and compositionality. Error type analysis of the second stage in Block 7 for the mismatch condition for in-lab participants, Mturk participants, the Option Model and the Task-Set Model.

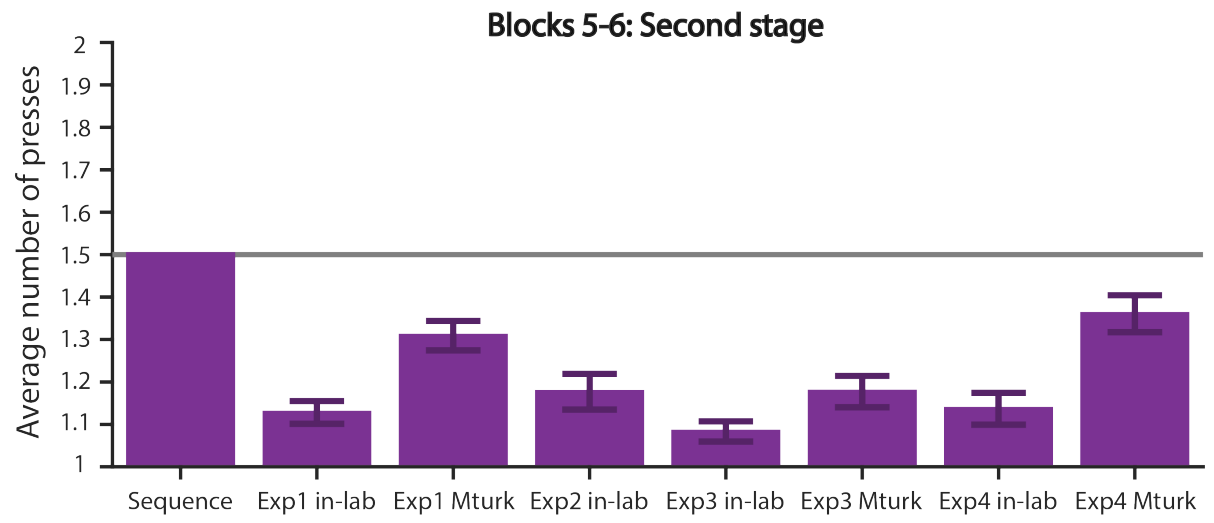

Supplementary Figure S16: Comparison of sequence learning model asymptotic performance with participants' performance in the last 10 trials of Blocks 5 and 6 across all 7 datasets (4 in-lab and 3 Mturk). While the sequence learning model is stuck at 1.5 presses/trial on average, participants performed significantly better. 

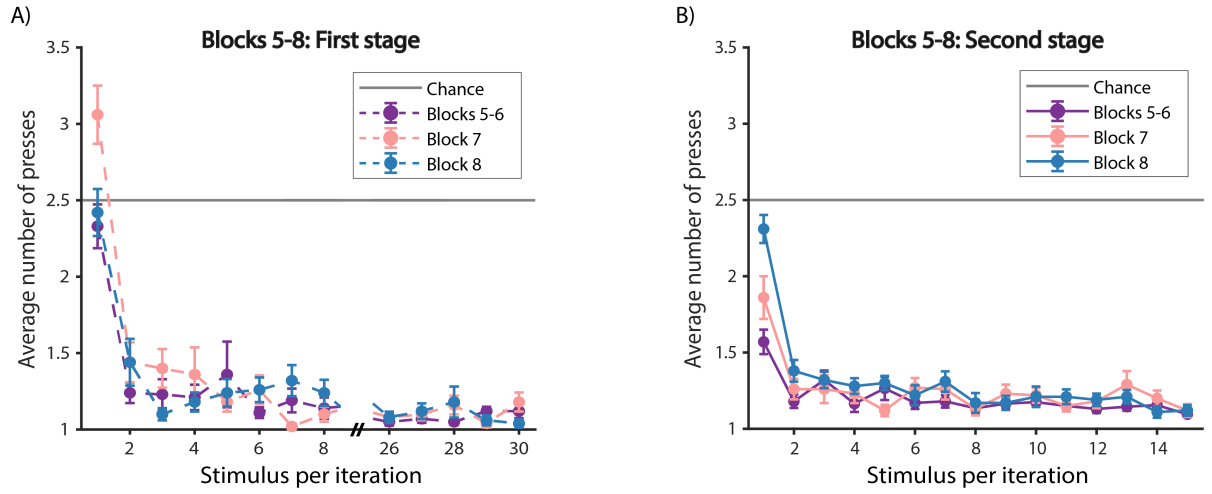

Supplementary Figure S17: Experiment 1 performance within Blocks 5-8 for in-lab participants. (A) First stage. (B) Second stage.

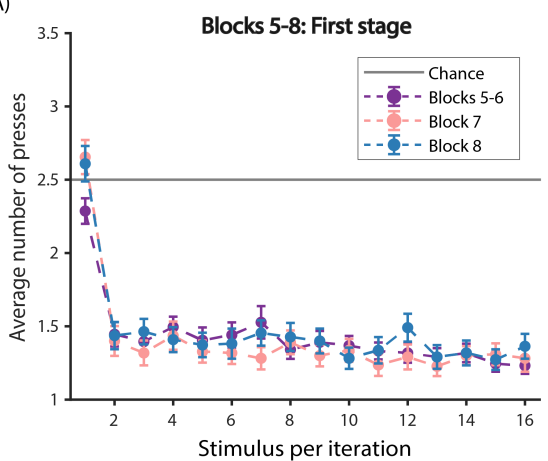

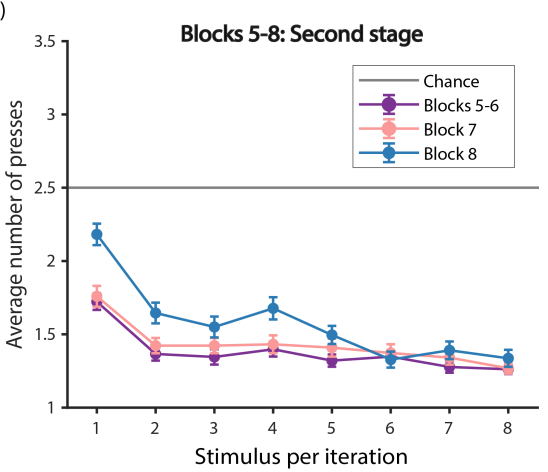

Supplementary Figure S18: Experiment 1 performance within Blocks 5-8 for Mturk participants. (A) First stage. (B) Second stage. 

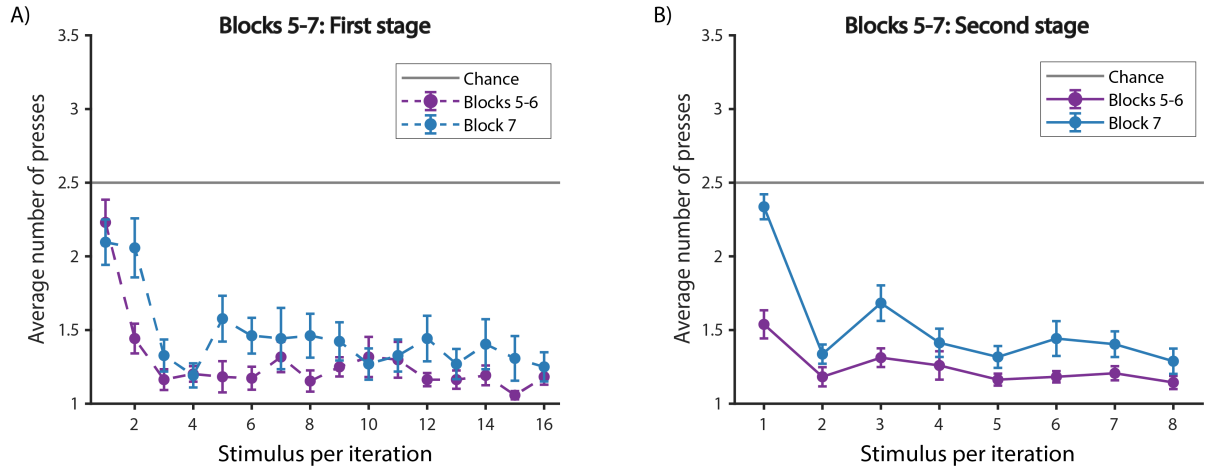

Supplementary Figure S19: Experiment 2 performance within Blocks 5-7. (A) First stage. (B) Second stage.
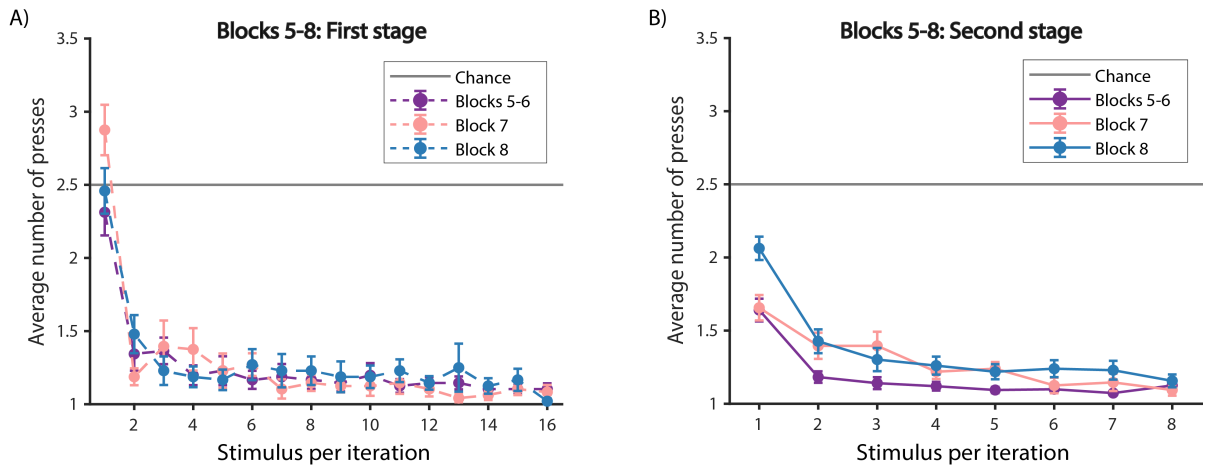

Supplementary Figure S20: Experiment 3 performance within Blocks 5-8 for in-lab participants. (A) First stage. (B) Second stage. 
A)

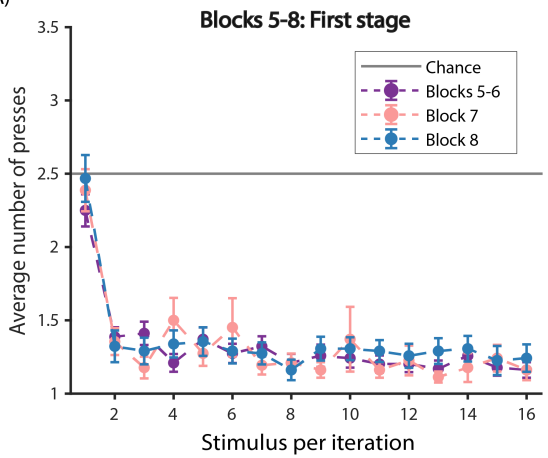

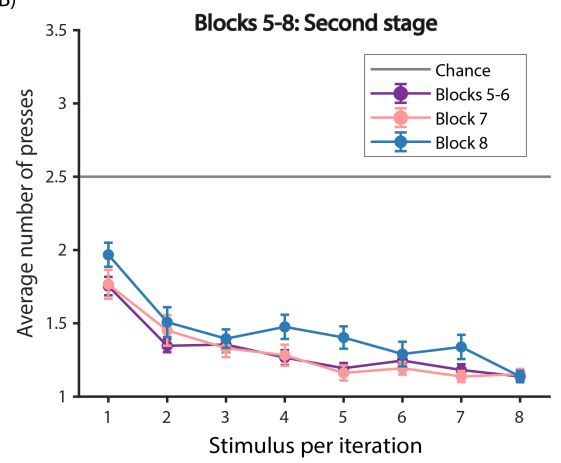

Supplementary Figure S21: Experiment 3 performance within Blocks 5-8 for Mturk participants. (A) First stage. (B) Second stage.

A)

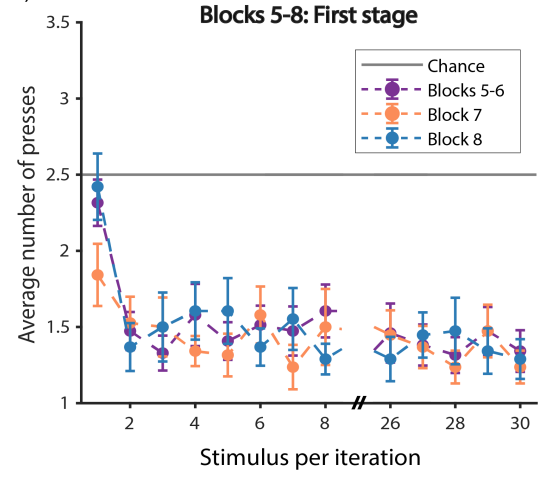

B)

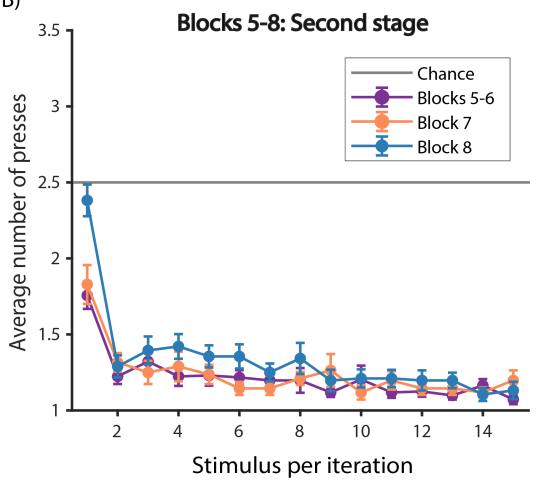

Supplementary Figure S22: Experiment 4 performance within Blocks 5-8 for in-lab participants. (A) First stage. (B) Second stage. 

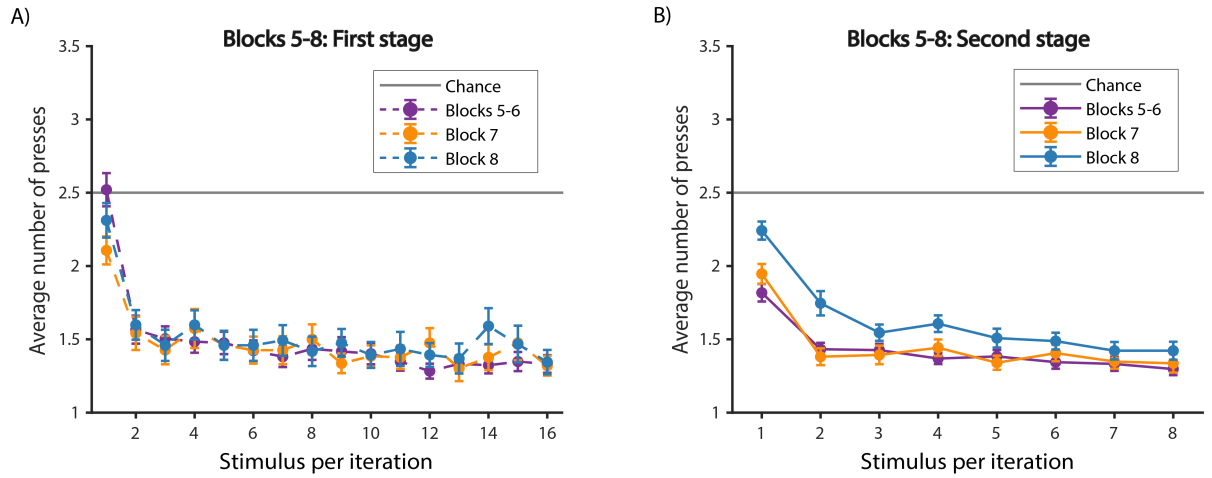

Supplementary Figure S23: Experiment 4 performance within Blocks 5-8 for Mturk participants. (A) First stage. (B) Second stage.

\begin{tabular}{|c|c|c|c|c|c|c|c|}
\hline Exp & $18-25$ & $26-30$ & $31-35$ & $36-40$ & $41+$ & Unknown & Total \\
\hline Exp 1 & 14 & 18 & 26 & 23 & 33 & 2 & 116 \\
\hline Exp 3 & 4 & 9 & 18 & 9 & 25 & 0 & 65 \\
\hline Exp 4 & 14 & 17 & 24 & 15 & 40 & 0 & 110 \\
\hline
\end{tabular}

Supplementary Table S3: Age range distribution for Mturk participants in Experiments 1,3 , and 4 . 